\title{
THE GALACTIC MAGNETIC FIELD DERIVED FROM COSMIC-RAY ELECTRONS
}

\author{
H. OKUDA* and Y. TANAKA**
}

Received 9 July 1968

An estimate of the galactic magnetic field is obtained by combining new results on the cosmic-ray electron spectrum with recent radio data. The lower and upper limits of the magnetic field in the galactic disk, derived from two alternative models of

\section{Introduction}

A number of estimates of the galactic magnetic field, based either on observational data or on theoretical considerations, have been published. Among the various methods that have been proposed, combination of the non-thermal radio emission with data on cosmicray electrons provides a suitable method for estimating a large-scale magnetic field. Since this method was first introduced (BIERMANN and DAVIS, 1960), the observational data on the radio spectrum as well as on the cosmic-ray electrons have become much more accurate and reliable. In the present work a new estimate of the magnetic field strength in the galactic disk is obtained with the aid of these data.

\section{The synchrotron radiation}

The non-thermal radio emission is interpreted as due to relativistic electrons moving in the interstellar magnetic field. This process is fully described by the theory of synchrotron radiation (cf. GINZBURG, 1958).

If the cosmic-ray electrons have an energy spectrum of the form

$$
\begin{aligned}
& N(E) \mathrm{d} E=N_{\mathrm{e}} E^{-\gamma} \mathrm{d} E \\
& \quad \mathrm{~cm}^{-2} \cdot \mathrm{sec}^{-1} \cdot \operatorname{sterad}^{-1} \cdot \mathrm{GeV}^{-1},
\end{aligned}
$$

the volume emissivity of synchrotron radiation $\varepsilon(v)$ is determined by

$$
\begin{gathered}
\varepsilon(v)=5.43 \times 10^{-32} U(\gamma)\left(4.64 \times 10^{6}\right)^{\gamma-1} N_{\mathrm{e}} H_{\perp}{ }^{(\gamma+1) / 2} \\
v^{-(\gamma-1) / 2} \mathrm{erg} \cdot \mathrm{cm}^{-3} \cdot \mathrm{sec}^{-1} \cdot \operatorname{sterad}^{-1} \cdot \mathrm{Hz}^{-1},
\end{gathered}
$$

\footnotetext{
* Present address: Kyoto University, Kyoto, Japan. ** Present address: Nagoya University, Nagoya, Japan.
}

field configuration, are $(0.5-1.0) \times 10^{-5} \mathrm{G}$ near the solar system and $(1.0-2.0) \times 10^{-5} \mathrm{G}$ near the galactic centre. The magnetic field in the halo is estimated to exceed $2.5 \times 10^{-6} \mathrm{G}$.

where $H_{\perp}=H \sin \theta$ is the magnetic field component (in Gauss) perpendicular to the line of sight, $\theta$ is the angle between the field and the line of sight, and $U(\gamma)$ a slowly varying function of $\gamma$. With $\gamma=2.4$ we have $U(2.4)=0.105$. Thus, for given $\varepsilon(v)$ and the electron spectrum the magnetic field $H_{\perp}$ can be obtained from eq. (2).

\section{The observational data}

The energy spectrum of cosmic-ray electrons measured by several groups (BLEEKER et al., 1967; DANIEL and Stephens, 1966; L'Heureux and Meyer, 1967; WeBBER and СноткоwSKI, 1967) is shown in figure 1. A significant change of the slope is observed around 1 to $2 \mathrm{GeV}$. Although most of the observations were made in the period of minimum solar activity, this effect may possibly be due to residual solar modulation. However, there is some evidence indicating the absence of solar modulation effect for cosmic-ray electrons (L'Heureux et al., 1967; BLEEKER et al., 1967). In the following discussion we assume, therefore, that the cosmic-ray electrons in interstellar space have the same spectrum as that presented in figure 1, and are distributed uniformly throughout the Galaxy.

In the energy range between 2 and $10 \mathrm{GeV}$ this spectrum can be approximated by

$$
\begin{aligned}
N(E)= & 8 \times 10^{-3} E^{-2.4} \\
& \mathrm{~cm}^{-2} \cdot \mathrm{sec}^{-1} \cdot \operatorname{sterad}^{-1} \cdot \mathrm{GeV}^{-1} .
\end{aligned}
$$

The value of the exponent, $\gamma=2.4$, gives a radio spectral index $\alpha=(\gamma-1) / 2=0.7$, which is in agreement 
with the values determined from recent radio observations for the frequency range above $85 \mathrm{MHz}$ (PURTON, 1966; WIELEBINSKI, private communication).

Several surveys of the non-thermal radio emission have been made with sufficiently narrow beamwidths to resolve the galactic disk. Here we adopt the surveys at 85.5 (Hill et al., 1958), 408 (LARGE et al., 1961), and $1440 \mathrm{MHz}$ (Mathewson et al., 1962). In the last two papers the non-thermal component was separated from the thermal one by the method introduced by WESTERHOUT (1958). The survey at $404 \mathrm{MHz}$ with a larger beamwidth $\left(7^{\circ} .5\right)$ (PaulinY-Toth and Shakeshaft, 1962 ) is also included to cover the anti-centre region.

The longitudinal variation of the non-thermal component in the galactic plane $\left(b^{\mathrm{II}}=0^{\circ}\right)$ is reproduced in figure 2 . The brightness temperatures observed at different frequencies were converted to values at $404 \mathrm{MHz}$

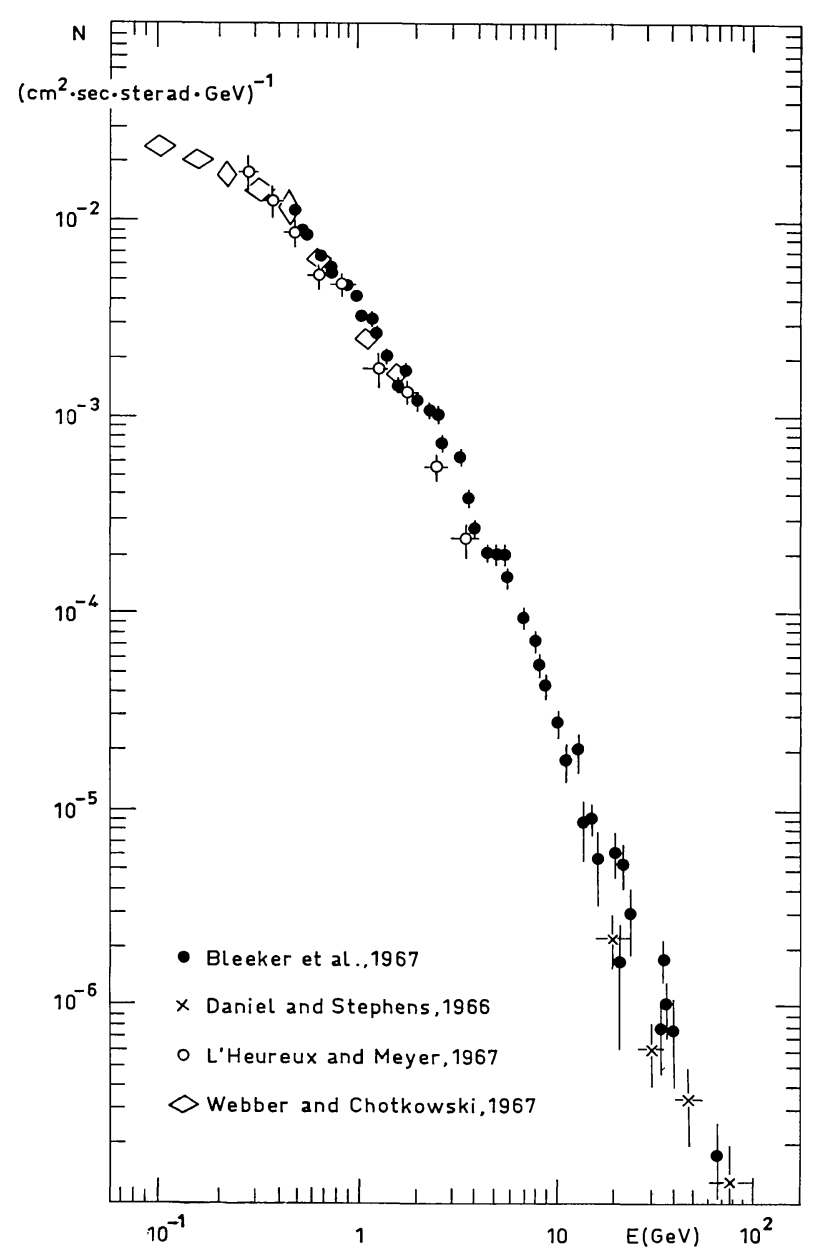

Figure 1. The observed energy spectrum of cosmic-ray electrons.

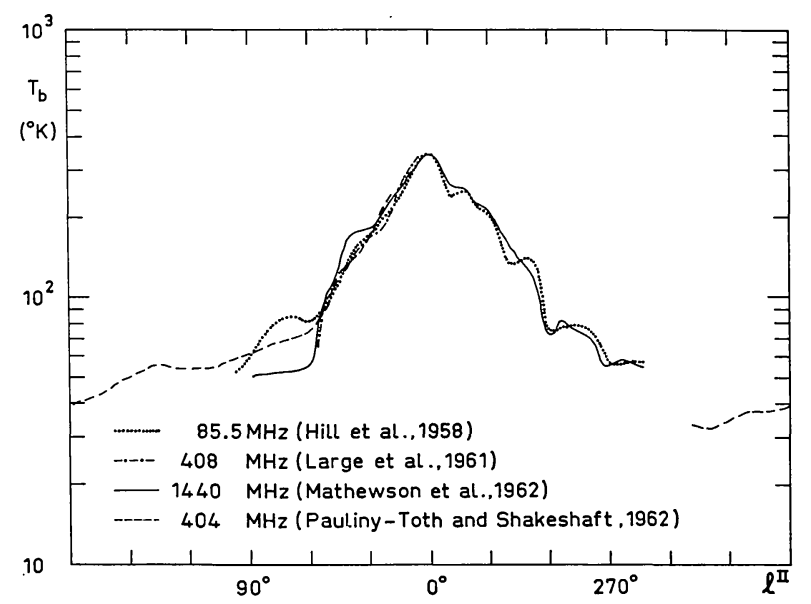

Figure 2. The longitudinal variation of the non-thermal radio component in the galactic plane. The observed brightness temperatures have been converted to values at $404 \mathrm{MHz}$ according to $v^{-2.7}$.

on the assumption that the brightness temperature is proportional to $v^{-2.7}$ as derived from eqs. (2) and (3). The agreement among these surveys is satisfactory. Average values are used in the following estimates.

Suppose we draw a circle around the galactic centre through the solar system. If the emissivity distribution in the disk is assumed to be axially symmetric about the galactic centre, the average volume emissivity along the line of sight inside this circle (the inner part of the Galaxy) at a given longitude $l^{\mathrm{II}}$ can be calculated by

$$
\left\langle\varepsilon\left(l^{\mathrm{II}}\right)\right\rangle=\left[T\left(l^{\mathrm{I}}\right)-T\left(l^{\mathrm{I}}+180^{\circ}\right)\right] / 2 R_{0} \cos l^{\mathrm{II}},
$$

where $R_{0}=10 \mathrm{kpc}$ is the distance between the Sun and the galactic centre. The quantity $\left\langle\varepsilon\left(l^{\mathrm{IL}}\right)\right\rangle$ obtained from the data of figure 2 is plotted against $l^{\mathrm{II}}$ in figure 3 . The average emissivity strongly increases toward the direction of the galactic centre.

\section{The magnetic field in the galactic disk}

Two alternative models of the magnetic field configuration in the disk are considered here. Both models can explain the radio observations.

Model 1: The magnetic field strength is constant over the entire disk, and the lines of force wind uniformly around the galactic centre. In this case the longitudinal variation of the emissivity is determined by the relation $\varepsilon \propto(\sin \theta)^{(\gamma+1) / 2}$ [see eq. (2)]. If a mean angular devia- 


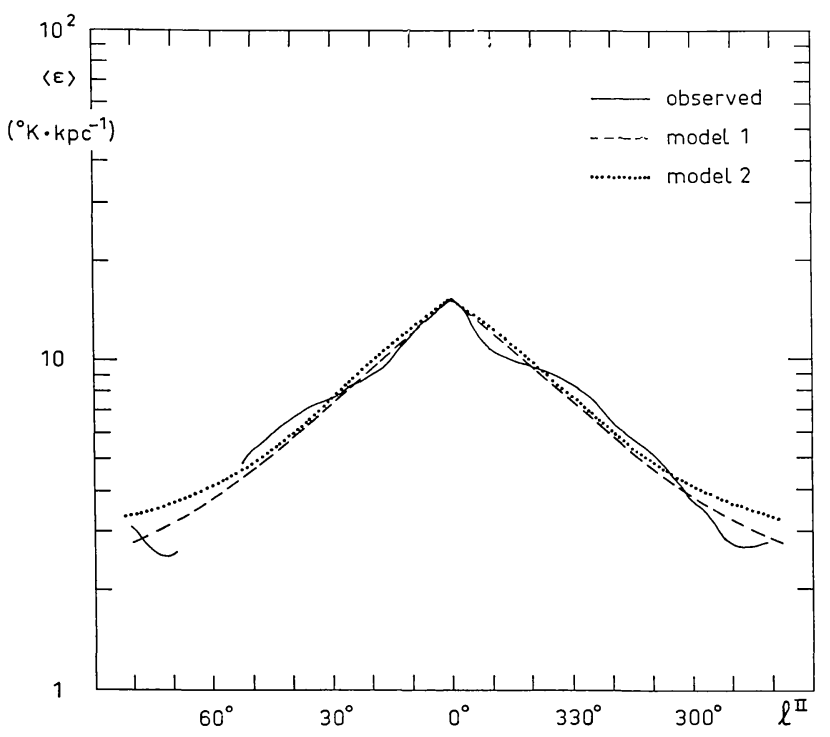

Figure 3. The average volume emissivity along the line of sight in the inner part of the Galaxy as a function of galactic longitude. The curves have been normalized at $l^{\mathrm{II}}=0^{\circ}$.

tion of $20^{\circ}$ from the circle is introduced, either systematic or random, the result agrees with the observation as shown by the broken line in figure 3 .

Model 2: The orientation of the magnetic field is completely random and, therefore, the emission is isotropic. The magnetic field strength, and hence the volume emissivity, increases toward the galactic centre. A volume emissivity of the form

$$
\varepsilon(R)=\varepsilon_{0} \exp \left(-2.5 R / R_{0}\right),
$$

where $R$ is the distance from the centre, gives a good agreement with the observations. This is indicated by the dotted curve in figure 3.

We have estimated for these two models, what magnetic field strength in the galactic disk must be introduced into eq. (2) in order to make the match shown in figure 3 quantitatively correct. The results are as follows:

Model 1 (winding field of uniform strength)

$$
\langle H\rangle=1.1\left\langle H_{\perp}\right\rangle=1.0 \times 10^{-5} \mathrm{G} ;
$$

Model 2 (randomly oriented field)

$\langle H\rangle=1.24\left\langle H_{\perp}\right\rangle=2.0 \times 10^{-5} \exp \left(-1.5 R / R_{0}\right) \mathrm{G} ;$

hence $\langle H\rangle=2.0 \times 10^{-5} \mathrm{G}$ near the galactic centre, and $\langle H\rangle=5 \times 10^{-6} \mathrm{G}$ near the solar system.
It can be shown that the values obtained from these two extreme models in eqs. (6) and (7) essentially give the lower and the upper limits for the strength of the large-scale magnetic field in the disk. The possible range of values thus found is:

$$
\begin{aligned}
& \text { near the solar system }(0.5-1.0) \times 10^{-5} \mathrm{G}, \\
& \text { near the galactic centre }(1.0-2.0) \times 10^{-5} \mathrm{G}
\end{aligned}
$$

The actual situation appears to be intermediate between models 1 and 2. This fact is borne out if we apply these two models to the anti-centre region in order to account for the observed brightness temperature of $40{ }^{\circ} \mathrm{K}$ at $404 \mathrm{MHz}$ (see figure 2). Model 1 results in a distance of $2 \mathrm{kpc}$ from the Sun to the outer boundary of the disk, which seems rather too short. Model 2 gives a brightness temperature of $15^{\circ} \mathrm{K}$, which is too low, even if the integration of (5) is extended to infinite distance.

In the preceding discussion we assumed a uniform distribution of cosmic-ray electrons in the galactic disk. However, the observed longitudinal variation of the radio emission (figure 3 ) can also be explained in terms of a larger concentration of cosmic-ray electrons towards the galactic centre. The following consideration may be relevant in assessing this alternative.

The energy density of cosmic rays in the vicinity of the Earth is about $1 \mathrm{eV} \cdot \mathrm{cm}^{-3}$. This is equal to an energy density of the magnetic field of $0.6 \times 10^{-5} \mathrm{G}$, comparable with the value near the solar system estimated above. This fact may be considered as an equilibrium condition for the confinement of cosmic rays in the magnetic field. If this equipartition of energy is assumed to hold near the galactic centre as well, the cosmic-ray intensity there could be as much as four times the value observed near the Earth, and the equilibrium magnetic field turns out to be $1.2 \times 10^{-5} \mathrm{G}$, provided the fraction of electrons in the cosmic-ray particles is constant everywhere.

It is unlikely that the cosmic-ray intensity near the galactic centre is much greater than this limit. Otherwise, the cosmic-ray energy density would largely exceed that of the magnetic field, since a larger electron intensity necessitates a weaker magnetic field in order to explain the given radio emission. Such a state would not be compatible with a stable confinement of cosmic rays. 


\section{The magnetic field in the halo}

Large ambiguities are present in the characteristics of the halo as compared with the disk. In particular, the dimension of the halo, the distribution of cosmicray electrons, and the contribution of the extragalactic radio component, are uncertain. We tentatively assume that the thickness of the halo in the polar direction is $10 \mathrm{kpc}$ at the position of the Sun, and that the cosmicray electrons are distributed uniformly in the halo with the same intensity as in the disk [eq. (3)].

The observed brightness temperature at $404 \mathrm{MHz}$ in the polar direction is about $20^{\circ} \mathrm{K}$ (PAULinY-Toth and SHAKeSHAFT, 1962). If the half-width of the radio disk is assumed to be $0.2 \mathrm{kpc}$, the contribution of the disk component in this direction is about 1 to $3{ }^{\circ} \mathrm{K}$ depending on whether model 2 or 1 is used. The contribution of the extragalactic component has been estimated to be of the order of 30 per cent or less (SHAIN, 1959; TURTLE et al., 1962; YATES and WieleBINSKI, 1966). Thus we take $12{ }^{\circ} \mathrm{K}$ for the halo contribution. Assuming a random magnetic field, we obtain

$$
\langle H\rangle_{\text {balo }}=2.5 \times 10^{-6} \mathrm{G} \text {. }
$$

Several estimates of the halo magnetic field under similar assumptions have recently been published and give values in the same range as above (SIRONI, 1965; FeLTEN, 1966).

It seems probable, however, that the average density of cosmic-ray electrons in the halo would be lower than that in the disk. Moreover, a smaller halo extension than that assumed here, seems more likely. On account of these two effects, the actual value of the field in the halo may well be larger than $2.5 \times 10^{-6} \mathrm{G}$.

There appears to be good evidence that the radio spectral index increases with increasing frequency (Yates and Wielebinski, 1966; YATES, 1966). A numerical calculation of the radio spectrum in the direction of the halo has been carried out (ОKUDA, 1968) starting from the observed spectrum of cosmic-ray electrons as presented in figure 1 . The good agreement between the results of this machine computation and the observed radio spectrum in the frequency range $10-400 \mathrm{MHz}$ supports the view that the value of the halo magnetic field estimated above is not far from the reality, and that the interstellar spectrum of cosmic-ray electrons has a bend around 1 to $2 \mathrm{GeV}$ similar to that in figure 1 . This bend accounts for the observed curvature of the radio spectrum (TANAKA, 1967).

\section{Acknowledgements}

This work was done while one author (Y.T.) held a position with the "Werkgroep Kosmische Straling" of the Netherlands Committee for Geophysics and Space Research, while the other author (H.O.) held a position with Leiden Observatory. We are indebted to Professor H. C. van de Hulst for advice and wish to thank Mr. W. N. Brouw and Miss E. M. Berkhuijsen for useful discussions.

\section{References}

L. BiermanN and L. Davis, 1960, Z. Ap. 5119

J. A. M. Bleeker, J. J. Burger, A. J. M. Deerenberg, A. Scheepmaker, B. N. Swanenburg and Y. Tanaka, 1968, Proc. Tenth Int. Conf. Cosmic Rays, Canadian J. Phys. 46522

R. R. Daniel and S. A. Stephens, 1966, Phys. Rev. Letters 17935 J. E. Felten, 1966, Ap. J. 145589

V. L. Ginzburg, 1958, Progress in Elementary Particles and Cosmic Ray Physics (North-Holland Pub. Co., Amsterdam) IV 335

J. L'Heureux and P. Meyer, 1968, Proc. Tenth Int. Conf. Cosmic Rays, Canadian J. Phys. 46892

J. L'Heureux, P. Meyer, S. D. Verma and R. Vogt, 1968, Proc. Tenth Int. Conf. Cosmic Rays, Canadian J. Phys. 46896

E. R. Hill, O. B. Slee and B. Y. Mills, 1958, Australian J. Phys. 11530

M. L. Large, D. S. Mathewson and C. G. T. Haslam, 1961, Mon. Not. Roy. Astr. Soc. 123113

D. S. Mathewson, J. R. Healey and J. M. Rome, 1962, Australian J. Phys. 15354

H. OKuda, 1968, Bull. Astr. Inst. Netherlands 19352

I. I. K. Pauliny-Toth and J. R. Shakeshaft, 1962, Mon. Not. Roy. Astr. Soc. 12461

C. R. Purton, 1966, Mon. Not. Roy. Astr. Soc. 133463

C. A. ShaIn, 1959, Symp. I.A.U. 9328

G. Sironi, 1965, Nuovo Cimento Ser. X 39372

Y. Tanaka, 1968, Proc. Tenth Int. Conf. Cosmic Rays, Canadian J. Phys. 46536

A. J. Turtle, J. F. Pugh, S. Kenderdine and I. I. K. PaulinyToth, 1962, Mon. Not. Roy. Astr. Soc. 124297

W. R. Webber and C. Chotkowski, 1967, J. Geophys. Res. 72 2783

G. Westerhout, 1958, Bull. Astr. Inst. Netherlands 14215

K. W. YATES, 1966, preprint (Univ. of Sydney)

K. W. Yates and R. Wielebinski, 1966, Australian J. Phys. 19 389 